\title{
Phase-Change Materials; the Challenges for TEM
}

Shalini Tripathi ${ }^{1}$, Matthew Janish ${ }^{2}$, Faruk Dirisaglik ${ }^{1}$, Adam Cywar ${ }^{1}$, Yu Zhu ${ }^{3}$, Katie Jungjohann ${ }^{4}$, Helena Silva ${ }^{1}$ and C. Barry Carter $^{2,5}$

${ }^{1 .}$ Electrical \& Computer Engineering, University of Connecticut, Storrs, CT.

${ }^{2 .}$ Materials Science \& Engineering, University of Connecticut, Storrs, CT.

3. IBM Thomas J Watson Research Center, 1101 Kitchawan Road, Yorktown Heights, NY.

4. Center for Integrated Nanotechnologies (CINT), Sandia National Lab, Albuquerque, NM.

5. Chemical \& Biomolecular Engineering, University of Connecticut, Storrs, CT.

Phase-change memory (PCM) materials are being developed for faster, non-volatile and high-density memory devices for more efficient computation and data storage. These materials are chalcogenides that can be switched between amorphous and crystalline phases producing orders of magnitude difference in electrical resistivity $[1,2]$. Large-scale use of PCM has been hindered by the large power required to heat the nano-scale element above crystallization or melting (for melt-quench amorphization) and by limited reliability due to resistance drifts of the metastable phases, elemental segregation and void formation upon extensive cycling. If these device characteristics can be improved, PCM can be integrated on top of CMOS as large-scale non-volatile on-chip storage, leading to significant performance and energy improvements in memory intensive applications by practically eliminating memory access latencies and refresh operations. In this work we are using TEM to study the phase changes that occur in these materials and devices with the aim of relating microstructure findings with results from electrical characterization and models to improve understanding of these materials and in turn enable design of improved devices.

The experiments involve thin film materials and devices fabricated on silicon substrates as well as thin films deposited directly onto Protochips carriers. Use of the Protochips heating holders is critical to achieve highly controlled temperature changes while imaging in the TEM. Although the rate of temperature change that can be achieved is too slow compared to PCM device operation it can still provide valuable insights into the various phase transformations and changes in the material and allow observation of other critical processes such as void formation, grain evolution and resistance drifts of the amorphous and crystalline fcc phases. Materials characterization uses both a Tecnai F30 and Titan ETEM which can accept the same Protochips heating holders. The ETEM is equipped with a K2 direct electron detector camera allowing high-speed video recording (up to $1600 \mathrm{frames} / \mathrm{s}$ ) of the structural changes that occur in these materials upon heating and cooling.

Figure 1a shows the morphology of the as-deposited film, which has the uniform gray contrast typical of amorphous material. The diffraction pattern in Figure $1 \mathrm{~b}$ confirms the lack of crystallinity in the film; the broad rings are typical of amorphous material. Figure 1c is a bright-field TEM image that shows the change in the GST film after the temperature was raised to $125^{\circ} \mathrm{C}$. The corresponding diffraction pattern is shown in Figure 1d, and is characteristic of polycrystalline material. The grains range in size, with some less than $10 \mathrm{~nm}$ in diameter and others as much as $75 \mathrm{~nm}$ across. Figure 2 shows three highmagnification images of the PCM film at room temperature after it had been heated ex-situ to $400^{\circ} \mathrm{C}$. The small crystallite have similar sizes but vary in orientation. In this case, irradiation by the electron beam at room temperature did not apparently change the particles. It is emphasized that comparing specimens that have been heat-treated in the TEM with those treated under typical processing 
conditions is critically important. This point will be explored and illustrated throughout this presentation [3].

\section{References:}

[1] S Fong, C Neumann, H Wong, IEEE Trans Electron Devices 64 (2017), p. 4374.

[2] D Lencer, M Salinga, M Wuttig, Advanced Materials 23 (2011), p. 2030.

[3] ST, HS and CBC acknowledge the funding support by the National Science Foundation under award DMR-1710468. The TEM analysis was performed at the Center for Integrated Nanotechnologies, an Office of Science User Facility operated for the U.S. Department of Energy (DOE) Office of Science by Los Alamos National Laboratory (Contract DE-AC52-06NA25396) and Sandia National Laboratories (Contract DE-NA-0003525).
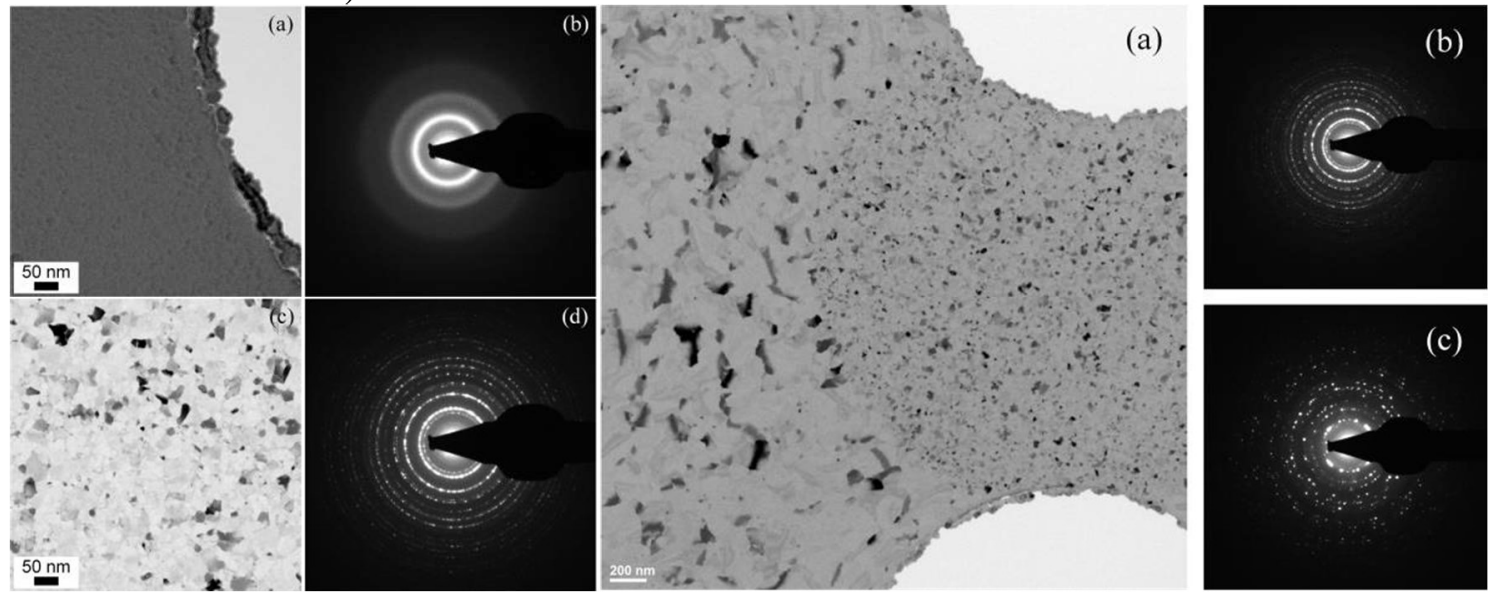

Figure 1. Left: a, b) Morphology of the as-deposited amorphous film and corresponding diffraction pattern with broad rings before heating. The chuck was then heated from RT to $100{ }^{\circ} \mathrm{C}$ at $0.5{ }^{\circ} \mathrm{C} / \mathrm{s}$ and then to $125^{\circ} \mathrm{C}$ at $0.1{ }^{\circ} \mathrm{C} / \mathrm{s}$. Crystallization began at $\sim 115^{\circ} \mathrm{C}$ and no changes were observed after $80 \mathrm{~s}$. The film was held at $125{ }^{\circ} \mathrm{C}$ for 10 minutes, then cooled to RT at $0.5{ }^{\circ} \mathrm{C} / \mathrm{s}$ at which point the "after" images were recorded. c, d) Bright-field image and the corresponding diffraction pattern of the annealed poly-crystalline material. Right: (a) Low-magnification bright- field image showing the electron beam irradiated area with smaller grain size. $(b, c)$ Diffraction patterns from the region with the smaller and larger grains, with same d-spacing. The white circular regions are holes in the film.

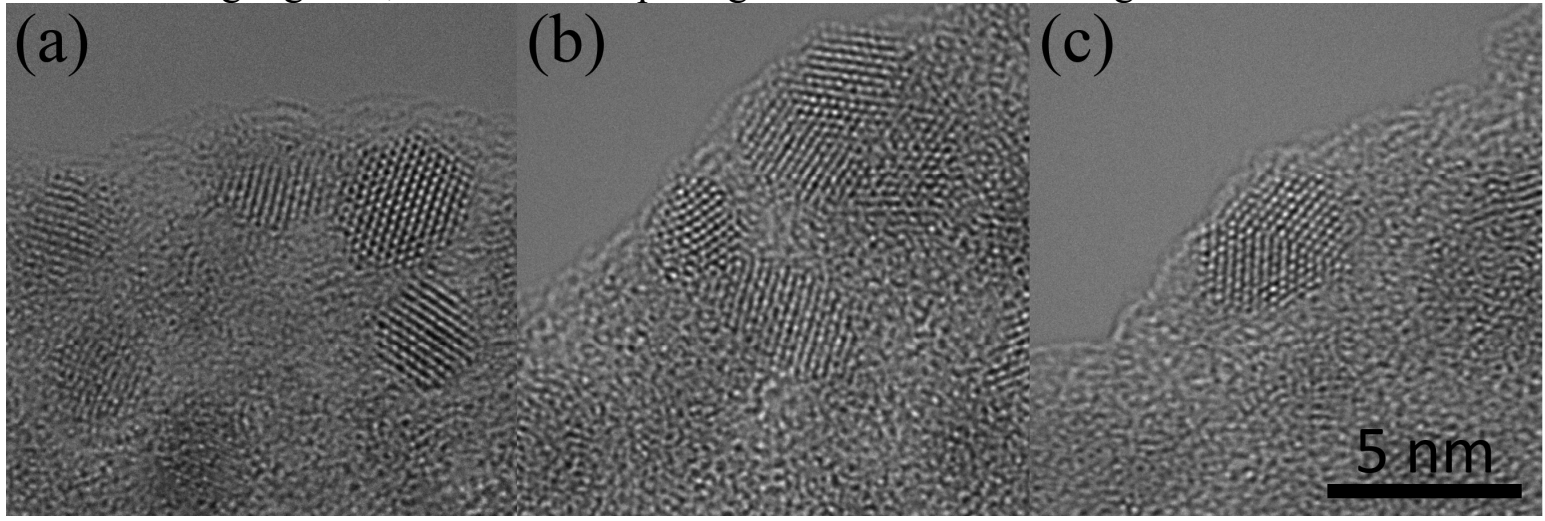

Figure 2. a-c: Three high-resolution images of a GST film after ex-situ heating at $400^{\circ} \mathrm{C}(300 \mathrm{kV}$ Csimage-corrected Titan). The grains are typically $\sim 3 \mathrm{~nm}$ in diameter and may contain twin boundaries. No change in size was observed during observation in the TEM. 